\title{
Esmalte en Terminología: Concordancia y Propuesta para Terminologia Anatomica, Histologica y Embryologica
}

\author{
Enamel in Terminology: Concordance and Proposal for \\ Anatomical, Histological and Embryological Terminology
}

Camila Panes $^{1}$; Christopher Nicholson ${ }^{2} \&$ Mariano del Sol $^{3}$

\begin{abstract}
PANES, C.; NICHOLSON, C. \& DEL SOL, M. Esmalte en Terminología: Concordancia y propuesta para Terminologia Anatomica, Histologica y Embryologica. Int. J. Morphol., 38(3):670-676, 2020.

RESUMEN: El esmalte dental es una estructura considerada en Terminologia Anatomica, Histologica y Embryologica. Los términos incluidos relacionados a él presentan tres raíces asociadas: enamel, amelo y adamantino. El objetivo de este artículo es analizar la etimología de estas raíces y presentar propuestas en latín y español que unifiquen las denominaciones relacionadas al esmalte dentario. En la búsqueda fueron utilizaron diccionarios médicos, terminológicos y etimológicos. Los resultados obtenidos señalan tres orígenes diferentes para cada raíz: adamantino es un término grecolatino relacionado a la extrema dureza de una estructura; enamel tiene un origen anglo-normando y antiguo alto germánico relacionado a la acción de fundir, derretir, y el aspecto estético que esto produce en un material; y amelo, el cual en sí mismo está relacionado a la botánica mientras que el término completo ameloblasto, fue acuñado por un académico estadounidense. Si bien la etimología de adamantino y enamel señalan características de firmeza y estética, respectivamente, en el desarrollo de la propuesta se decidió mantener el término latinizado enamelum y su adjetivo enameleus, eliminando las otras dos raíces relacionadas al esmalte dentario. Se realizó la propuesta para los cambios en los términos del idioma latín y su traducción al español, acorde a los requerimientos de la FIPAT para Terminologia Anatomica, Histologica y Embryologica, con el fin de unificar la denominación de los términos asociados y derivados del esmalte dental, para fines académicos y de investigación.
\end{abstract}

PALABRA CLAVE: Esmalte; Terminologia Anatomica, Histologica y Embryologica.

\section{INTRODUCCIÓN}

Durante 2019 y 2017 se realizó la publicación de la segunda edición de Terminologia Anatomica y Terminologia Embryologica respectivamente, disponible en el sitio web de la Federative International Programme for Anatomical Terminology (FIPAT). Si bien se mantienen las agrupaciones clásicas por sistemas, en esta versión de Terminologia Embryologica se creó un listado que incluyó tanto nuevos términos del ámbito del desarrollo normal, como anomalías congénitas y variaciones del crecimiento. Para ambos escritos, el listado de los términos ya no cuenta con un código sino con una numeración seriada y el término oficial sigue siendo escrito en latín, con sus equivalentes en idioma inglés según habla inglesa o norteamericana, sumando a su vez sinónimos encontrados tanto en términos latinos como ingleses, permitiendo de esta forma una traducción facilitada hacia el idioma vernáculo que sea requerido (FIPAT, 2017). Por ahora, Terminologia Histologica mantiene un código para cada estructura, con su nominación en latín y su equivalente en inglés.

Para el caso del esmalte dental, estructura cuya principal característica es ser el tejido más duro del cuerpo humano, es considerado en Terminologia ya que puede ser observado de forma macroscópica como la capa más externa del diente; microscópica, siendo una estructura de contenido mayormente inorgánico con incapacidad de ser regenerada; y también desde el punto de vista de su formación, siendo la amelogénesis un interesante capítulo de la formación dental.

En este contexto, si bien la Terminología Odontológica (TO) y Orobiológica (TOrobiol) están aún en desarrollo y con publicación pendiente, es significativo continuar avanzando en la precisión de los términos empleados, unificando conceptos en un lenguaje morfológico universal. Como señalaron Algieri et al. (2013), la uniformidad y corrección de este lenguaje, facilita el proceso de aprendizaje, permitiendo un mejor desarrollo en

\footnotetext{
${ }^{1}$ Doctorado en Ciencias Morfológicas, Facultad de Medicina, Universidad de La Frontera, Temuco, Chile.

${ }^{2}$ Núcleo de Idiomas, Facultad de Estudios Interdisciplinarios, Universidad Mayor, Temuco, Chile.

${ }^{3}$ Centro de Excelencia en Estudios Morfológicos y Quirúrgicos (CEMyQ), Universidad de La Frontera, Temuco, Chile.
} 
el ámbito académico y clínico, pero también siendo una valiosa herramienta para la investigación.

El objetivo de este trabajo fue analizar la etimología de los términos relacionados al esmalte dental presentes en Terminologia Anatomica, Histologica y Embryologica, contribuyendo con propuestas de su denominación en latín y su traducción al español.

\section{MATERIAL Y MÉTODO}

Primero fueron seleccionados los términos anatómicos, histológicos y embriológicos relacionados a la palabra esmalte en Terminologia Anatomica (TA), Histologica (TH) y Embryologica (TE) (Tabla I). Luego fue consultado el Diccionario de Términos Médicos de la Real Academia de Medicina, para conocer la definición de estos

Tabla I. Términos relacionados a Esmalte en Terminologia Anatomica, Histologica y Embryologica.

\begin{tabular}{|c|c|c|c|}
\hline Código & Término en Latín & Término en Inglés & Sinónimos \\
\hline TA - 938 & Enamelum & Enamel & Substantia adamantina \\
\hline H3.04.01.0.02011 & Enamelum & Enamel & \\
\hline H3.04.01.0.02012 & Enameloblastus, Ameloblastus & Ameloblast & \\
\hline H3.04.01.0.02016 & Crystallum hydroxyapatiti enameli & Hydroxyapatite crystal of enamel & \\
\hline H3.04.01.0.02017 & Processus enameloblasti & Ameloblast process & \\
\hline H3.04.01.0.02019 & Lamella enamelea & Enamel lamella & \\
\hline H3.04.01.0.02020 & Fusus enameli & Enamel spindle & \\
\hline H3.04.01.0.02021 & Fasciculus enameli & Enamel fascicle & \\
\hline H3.04.01.0.02022 & Junctio dentinoenameli & Dentino-enamel junction & \\
\hline H3.04.01.0.02023 & Linea incrementalis enamelea & Enamel incremental line & \\
\hline H3.04.01.0.02059 & Linea incrementalis enamelea & Incremental line & \\
\hline H3.04.01.0.02084 & Ectodontium & Ectodontium & \\
\hline H3.04.01.0.02089 & Organum enameleum & Enamel organ & \\
\hline H3.04.01.0.02093 & Epithelium enameleum externum & External enamel epithelium & \\
\hline Н3.04.01.0.02094 & Pulpa enamelea; Reticulum stellatum & Stellate reticulum & \\
\hline H3.04.01.0.02096 & Epithelium enameleum internum & Internal enamel epithelium & \\
\hline H3 .04.01.0.05012 & Enameloblastus; Ameloblastus & Ameloblast; Enameloblast & \\
\hline H3.04.01.0.02097 & Cuticula enameli & Enamel cuticle & \\
\hline H3 .04.01.0.02100 & Residuum epitheliale & Residual epithelium; Reduced enamel epithelium & \\
\hline H3.04.01.0.02103 & Junctio dentinoenamelea & Enamel dentine junction; Dentin enamel junction & \\
\hline TE - 2605 & Organum enameleum & Enamel organ & \\
\hline TE - 2606 & Lamina basalis enameli & Enamel basal lamina & \\
\hline TE - 2607 & Nodus enameleus & Enamel knot & \\
\hline TE - 2608 & Nodus enameleus primarius & Primary enamel knot & \\
\hline TE - 2609 & Nodus enameleus secundarius & Secondary enamel knot & \\
\hline TE - 2614 & Chorda enamelea & Enamel cord & \\
\hline TE - 2616 & Epithelium enameleum externum & External enamel epithelium & Outer enamel epithelium \\
\hline TE - 2617 & Pulpa enamelea & Stellate reticulum & Reticulum stellatum \\
\hline TE - 2618 & Stratum intermedium & Stratum intermedium & \\
\hline TE - 2619 & Epithelium enameleum internum & Internal enamel epithelium & Inner enamel epithelium \\
\hline TE - 2624 & Amelogenesis & Amelogenesis & \\
\hline TE - 2625 & Preameloblastus & Pre-ameloblast & \\
\hline TE - 2626 & Ameloblastus & Ameloblast & \\
\hline TE - 2627 & Amelogenesis & Amelogenesis & \\
\hline TE - 2629 & Prisma enameli & Enamel prism & Enamel rod \\
\hline TE - 2655 & Epithelium enameleum reductum & Reduced enamel epithelium & \\
\hline TE - 2656 & Cuticula enamelea primaria & Primary enamel epithelium & \\
\hline TE - 2657 & Vestigium epithelii enamelei & Rest of enamel epithelium & \\
\hline TE - 2668 & Adamantinoma & Adamantinoma & Ameloblastoma \\
\hline TE - 2676 & Enameloma & Enameloma & \\
\hline TE - 2682 & Opacitas enameli & Enamel opacity & \\
\hline TE - 2683 & Extensio cervicalis enameli & Cervical enamel extension & \\
\hline TE - 2696 & Hypoplasia enameli & Enamel hypoplasia & \\
\hline TE - 2701 & Amelogenesis imperfecta & Amelogenesis imperfecta & \\
\hline
\end{tabular}


términos y posibles derivados, raíz u origen (Tabla II). Los resultados obtenidos fueron buscados en diccionarios de latín (Sopena y Lewis \& Short), siendo consultados, además, diccionarios etimológicos del inglés, lenguas romances y germánico, buscando la etimología de las raíces encontradas (Tablas III y IV).

Tabla II. Resultados del Diccionario de Términos Médicos de la Real Academia de Medicina.

\begin{abstract}
Esmalte (fráncico *smalt 'esmalte' + -e esp.; docum. en fr. desde 1140 y en esp. desde 1291) [ingl. enamel] 1. s.m. [TA: enamelum] Tejido mineralizado que recubre la dentina en la zona de la corona y termina en el cuello del diente. Está formado por material inorgánico muy escaso (amelogeninas, enamelinas, ameloblastinas y tuftelinas) y por sales minerales. Es el tejido más duro del cuerpo humano. Sin.; capa adamantina, enamelo, esmalte dental, sustancia adamantina, tejido adamantino.

Adamantino, -na (lat. adamantin-u(m) del gr. adamántin-os/-ē ‘que no se doblega', ‘duro como el acero'; docum. en esp. desde 1500) [ingl. adamantine] 1. adj. Del esmalte dental o relacionado a él. Términos relacionados: adamantinoma $=$ ameloblastoma; adamantoblasto $=$ ameloblasto ; adamantoblastoma $=$ ameloblastoma .
\end{abstract}

Tabla III. Resultados de la búsqueda de términos relacionados a esmalte (enamel, amelo, adamantino y esmalte) en Diccionarios de latín-español y latín-inglés.

\title{
Diccionario Sopena
}

Ădămantēus, a, um. Adj. Ov. Véase adamantinus.

Ădămantĭnus, a, um. (de ădămas). Adj. Hor. de acero; acerado, acerino; duro como el acero.

Ădămantis, $\breve{l d i s . ~(d e l ~ g r . ~ a ~ p r i v . ~ y ~ d a m a o) . ~ f . ~ P l i n . ~ P l a n t a ~ h e r b a ́ c e a ~ d e l ~ A s i a ~ M e n o r, ~ a ~ l a ~ c u a l ~ s e ~ l e ~ a t r i b u i ́ a ~ l a ~}$ propiedad de domar a los leones, dulcificar la ferocidad, y resistir a todo esfuerzo sin quebrarse.

Ădămas [acus., adamanta]. (del gr. adamas). m. Plin., Manil. Diamante. (poét.) Ov., Virg., Prop. Hierro del más sólido, acero. Solido adamante columnae, Virg., columnas de hierro macizo (o de durísima piedra). (fig.) Ov. Carácter inexorable, inflexible. Voce tua posses adamanta movere, Ov., con tu voz podrías remover los corazones más duros.

Amella, ae. f. Serv. Véase amellus.

Amellus, i. m. Virg. Amelo (flor).

Enamel: lat. Encaustum, i. (del gr. ēgkaustos). n. Plin. Encausto, pintura al encausto. Encaustum o sacrum encaustum. Cod. Th., Cod. Just., tinta de púrpura (reservada para uso del emperador).

\section{Diccionario Lewis \& Short}

Ădămas, antis, m. (acc. Gr. adamanta, adamantas), = ỏoá $\mu a \varsigma$ (invincible),

I adamant, the hard est iron or steel; hence poet., for any thing inflexible, firm, lasting, etc. (first used by Verg.): porta adversa ingens solidoque adamante columnae, Verg. A. 6, 552; cf. Mart. 5, 11; adamante texto vincire, with adamantine chains, Sen. Herc. F. 807.-Trop. of character, hard, unyielding, inexorable: nec rigidos silices solidumve in pectore ferrum aut adamanta gerit, a heart of stone, Ov. M. 9, 615: lacrimis adamanta movebis, will move a heart of stone, id. A. A. 1, 659; so id. Tr. 4, 8, 45: voce tua posses adamanta movere, Mart. 7, 99: duro nec enim ex adamante creati, Sed tua turba sumus, Stat. S. 1, 2, 69. -

II The diamond: adamanta infragilem omni cetera vi sanguine hireino rumpente, Plin. 20, prooem. 1; 37, 4, 15, §55 sq.

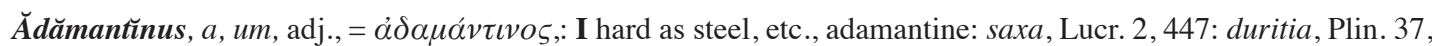
11, 73. - Hence poet., extremely hard, inflexible, invincible: clavi, Hor. C. 3, 24, 5: tunica, id. ib. 1, 6, 13: juga, Prop. 3, 9, 9; cf. aёnus.

Ădămantēus, a, um, adj. adamas,

I of hard steel, iron, etc., or hard as these: catenae, adamantine, Manil. 1, 921: nares (taurorum), Ov. M. 7, 104.

Ădămantis, ̌̆dis, f.,

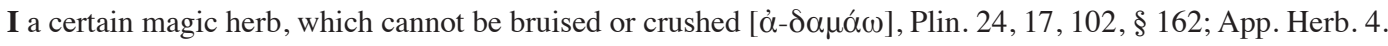

Amellus, i, m.,

I the purple Italian starwort: Aster amellus, Linn.; Verg. G. 4, 271 (cf. Serv. ad h. 1.); Col. 9, 4, 4.

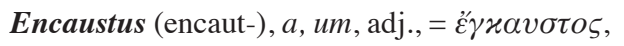

I burned in, encaustic.

I Adj.: genus pingendi, the encaustic mode of painting, Plin. 35, 11, 41, § 149; cf., respecting it, O. Müller, Archäol. § 320: Phaёthon, Mart. 4, 47, 1.-

II Subst.: en-caustum (encaut-), $i ., \mathrm{n} .,=\tilde{\varepsilon}^{\prime} \gamma x \alpha v \sigma \tau o v$, the purple-red ink of the later Roman emperors, Cod. Th. 7, 20, 1; August. contra Faust. 3, 18; Cod. Just. 1, 23, 6 al. 


\section{RESULTADOS}

De la búsqueda en Terminologia Anatomica, Histologica y Embryologica fue hallado solo 1 término en $T A, 19$ términos en $T H$ y 24 en $T E$. Todos ellos presentan la palabra esmalte, en el término en latín y/o en su traducción al inglés. Se incluyó el término "estrato intermedio", por ser parte del capítulo esmalte y encontrarse directamente relacionado a él.

De esta tabla resumen, podemos observar que existen términos repetidos dentro de $T H$ : Linea incrementalis enamelea (H3.04.01.0.02023; H3.04.01.0.02059); Enameloblastus, Ameloblastus (H3.04.01.0.02012; H3.04.01.0.05012); Junctio dentinoenameli (H3.04.01.0.02022) y Junctio dentinoenamelea (H3.04.01.0.02103). Además, existen 3 raíces asociadas a los términos de esmalte dental: enamel, amelo y adamantino, especialmente en $T E$ y $T H$.
La consulta en el Diccionario de Términos Médicos de la Real Academia de Medicina, detallada en la Tabla II, demuestra que si bien los términos adamantino y esmalte, tienen diferentes orígenes, se encuentran estrechamente relacionados debido a las características de dureza y resistencia que muestra el esmalte dental.

Posteriormente y teniendo en cuenta el origen grecolatino de adamantino, se realizó la búsqueda en dos diccionarios de latín (Tabla III). Se incluyó uno en idioma inglés, para eliminar posibles sesgos de idioma en las definiciones. Cabe destacar que enamelum, ameloblastus o enameloblastus, son términos latinizados que se encuentran indexados en terminología, y su búsqueda en diccionarios de latín no arroja resultados.

Con los resultados expuestos en la Tabla III, se puede entender la relación directa del esmalte dental, con la palabra de origen greco-latino ădămas y su adjetivo ădămantĭnus, debido a la característica resistencia, dureza

Tabla IV. Resultados de la búsqueda etimológica para los términos Adamantino y Enamel.

Oxford Concise Dictionary of English Etymology (1996)

Adamant: hard rock or mineral, now only as a symbol of extrem hardness (cf. Ezek. 3. 9); diamond; loadstone. - OF.- L. adamant - Gr. adamas, adamant, orig. adj. invincible, f. A + damás, The sense 'magnet, loadstone' arose rom assoc. of med. adamas with L. adamare have a strong liking for. Cf. diamond. So adamantine.

Enamel glass like composition laid on a Surface. xv. f. enamel vb xiv. -AN, enameler, enamailler. f. EN + amail - OF, esmail (mod. email). new formation on the nom esmauz - Gmc. *smalt (OHG smalz. G. schmalz melted (al). rel to smelt.

\section{Etymological Dictionary of the Romance languages (1864)}

Diamante It. Sp., Pr. diaman, Fr. diamant, E. diamond; from adamas adamantis, prob. influenced by diafano transparent. Another form is the Pr. adiman, aziman, aiman, O. Fr. aïmant, Fr. aimant, Sp. Pg. iman a magnet, in which sense L. L. adamas was used.

Smalto It., Wal. smaltz (jumaltz), Sp. Pg. esmalte, Fr. émail, E. enamel (from the frequent combination en émail, peindre en émail \&c.), L. L. smaltum. From O. H. G. smelzan smalzjan smaltjan, G. schmelzen, E. smelt. In the Fr. émail, $i$ is inserted after $a(e s m a i l l)$ and the $t$ dropt as in $g a l$ for $(/ \ll /$ i from G. wald).

\section{A Short Etymological Dictionary of Modern English (1966)}

Melt: (v, hence $\mathrm{n})$ - pt, pp (hence pa) melted—presp, hence pa and vn, melting; obs pp, now only adj, molten (OE gemolten); cf malt, $\mathrm{n}$, hence $\mathrm{v}$ (whence pa malted), maltose (malt, $\mathrm{n}+\mathrm{Chem}$-ose);-smelt, $\mathrm{v}$, whence smelter, smeltery, pa and vn smelting; cf smalt, whence smaltine, smaltite (Min suffixes -ine, -ite); prob milt, spleen; enamel.

1. 'To melt' derives from OE melt an, vi, sense-assiste by OE mieltan, myltan, vt: cf ON melta, to digest, Gr meldein (vt), to melt, and perh L mollis, soft: IE r, prob *meld-.

2. Akin to melt is smelt, to fuse (ore): MD or MLG smelten: cf OHG smelzan, MHG smelzen, G schmehen, to melt, vi, and the vt OHG-MHG smelzen, G schmelzen.

3. Akin to OE meltan, mieltan, to melt (vi, vt), is OE mealt, E malt, grain (usu barley) softened by steeping and thereupon allowed to germinate: $\mathrm{cf}$ ON malt, OHG-G malz (cf the OHG adj mah, melting away, and ON maltr, rotten), MD malt, molt, moud, D mout.

4. Akin to the $\mathrm{v}$ smelt is the $\mathrm{n}$ smalt, adopted from EF-F: It smalto, coloured glass, of Gmc origin: cf OHG smelzan (as in para 2), but esp OHG smelzi, G Schmelz, enamel, and cf MLG smalt, OHG-MHG smalz, G Schmalz, fat or lard, which melt easily and, in one form, result from cooking.

5. Enamel itself ( $\mathrm{v}$, hence $\mathrm{n}$ ) derives from AF enameler, earlier enamayller: en-, in(to) +amayl, OF esmail (F émail), earlier esmal, from a lost *esmalt: Frankish * smalt, deduced from OHG smelzi, enamel.

6. Akin prob to OE mealt, malt, and meltan, to melt, is OE milte, the spleen: cf Ofris milte, OHG milzi, MHG milze, G $\mathrm{MHz}, \mathrm{MD}$ melte, milte, D milt, ON milte, milti. 
e inflexibilidad de este tejido. Estas características también tienen un componente mitológico, ya que, como señala Robin Hard en el Gran Libro de la Mitología Griega, Gea, Diosa griega, crea una hoz de un material mítico de extrema dureza llamado Adamantio, con la finalidad de matar al Dios Urano.

La búsqueda del término esmalte desde el español al latín, sin embargo, genera mayor controversia, ya que no existe una relación aparente entre una forma de pintura, tinta o quemadura, con el esmalte dental o alguna de sus características. En la búsqueda etimológica, detallada en la Tabla IV, se utilizó la palabra en inglés enamel, para encontrar su relación no grecolatina del término, tal como refiere el origen de la palabra indicado en el Diccionario de Términos Médicos de la Real Academia Nacional de Medicina.

De los resultados expuestos en la Tabla IV, se observa la relación desde el Anglo-Normando y Alto Germánico Antiguo, hacia "melt", de derretir, fundir y su forma más cercana al esmalte mismo "smalt". Ameloblasto o su raíz, no registra en los diccionarios etimológicos consultados, más el término pudo ser rastreado hasta su acuñamiento en 1882 por William H. Eames (Diccionario Etimológico de la Universidad de Salamanca).

\section{DISCUSIÓN}

La elección de los términos anatómicos utilizados para comunicarnos universalmente debe ser actualmente acorde a los lineamientos dados por la FIPAT, siendo uno de los más relevantes el que los nombres de las estructuras deban tener un valor informativo y lo más descriptivo posible; suprimir los epónimos y homónimos y que los nombres de las estructuras derivadas de un término tengan congruencia y armonía entre sí en su denominación (Vásquez \& del Sol, 2015). Se sugiere, además, que en lo posible las estructuras sean denominadas con un solo término, que el idioma oficial empleado sea el latín y que la descripción del elemento sea fiel a los principios de geometría y forma de la estructura señalada.

Con los resultados antes expuestos, y la incierta asociación entre el smalt germánico, el enamayller fránces y enamel inglés, consideramos que esta estructura anatómica ha sido nombrada de tal forma por sus características estéticas, de especial translucidez y belleza, tal como si fuera una capa de vidrio fundido que recubre nuestros dientes. Esta posición se contrapone a la adoptada por los griegos y romanos, quienes consideraron a esta capa externa con una resistencia tal que la llamaron adamantio, mineral mitológico de extrema dureza.

Si bien ambas miradas de esta misma estructura tienen igual validez, debemos considerar el uso académico y científico de sus denominaciones. Una búsqueda inicial en el metabuscador PubMed, muestra que "enamel" cuenta con más de 19.000 menciones en artículos científicos, versus "ameloblast" y "amelogénesis", los cuales entre ambos solo tienen poco más de 2.600 publicaciones a la fecha. Esto hace difícil un cambio en su denominación.

La propuesta para las diferentes terminologías, se dividió en dos etapas, siendo en primer lugar necesario eliminar los términos repetidos en TH y suprimir dobles terminaciones (Tabla V).

Los términos que se sugieren mantener versus aquellos eliminados, se justifica en el hecho de que los primeros contienen mayor información o son más descriptivos en su traducción al inglés, o bien la terminología en latín es más exacta en su denominación.

En segundo lugar, se analizó el uso correcto del latín en los nombres resumidos en la Tabla I, teniendo en cuenta que se puede generar confusión entre enamelum (sustantivo) y enameleus (adjetivo). Parte de los cambios desarrollos como propuesta, incluyen la vinculación directa de un término mediante la adición de enamelum o su declinación correspondiente al nombre en latín de la estructura. Este fue el caso de Epithelium enamelum reductum $(\mathrm{H} 3.04 .01 .0 .02097)$ y Reticulum enameleum stellatum (H3.04.01.0.02094). Esto es de importancia pedagógica, ya que la relación directa mediante el nombre, facilita el aprendizaje, sobre todo en aquellos académicos y estudiantes que no se dedican al estudio de un solo tipo de tejido, y deben recordar nombres de todo el cuerpo.

Estos cambios realizados se basan fundamentalmente

Tabla V. Términos que deberían ser eliminados de Terminologia Histologica y Embryologica.

\begin{tabular}{ccc}
\hline Código & Término en Latín & Término en Inglés \\
\hline H3.04.01.0.02059 & Linea incrementalis enamelea & Incremental line \\
H3.04.01.0.02012 & Enameloblastus, Ameloblastus & Ameloblast \\
H3.04.01.0.02103 & Junctio dentinoenamelea & Enamel dentine junction; Dentin enamel junction \\
H3.04.01.0.02084 & Ectodontium & Ectodontium \\
2624 & Amelogenesis & Amelogenesis \\
\hline
\end{tabular}


debido la gran cantidad de artículos científicos en los cuales el inglés "enamel" es la principal forma de relacionar un término al esmalte dental. Es prioritario, además, unificar las denominaciones dadas a las estructuras en Terminologia Anatomica, Histologica y Embryologica, conectando así las publicaciones científicas relacionadas a un mismo órgano, tejido o proceso.

La propuesta desarrollada para Terminologia Anatomica, Histologica y Embryologica se exponen en la Tabla VI.

Tabla VI. Tabla con los términos relacionados a esmalte con los cambios propuestos.

\begin{tabular}{|c|c|c|c|}
\hline Código & Término en Latín & Término en Inglés & Propuesta en español \\
\hline TA - 938 & Enamelum & Enamel & Esmalte \\
\hline H3.04.01.0.02011 & Enamelum & Enamel & Esmalte \\
\hline H3.04.01.0.02016 & $\begin{array}{c}\text { Crystallum hydroxyapatiti } \\
\text { enameli }\end{array}$ & Hydroxyapatite crystal of enamel & $\begin{array}{c}\text { Cristal de hidroxiapatita del } \\
\text { esmalte }\end{array}$ \\
\hline H3.04.01.0.02017 & Processus enameloblasti & Enameloblast process & Proceso enameloblástico \\
\hline H3.04.01.0.02019 & Lamella enamelea & Enamel lamella & Laminilla del esmalte \\
\hline H3.04.01.0.02020 & Fusus enameli & Enamel spindle & Huso del esmalte \\
\hline H3.04.01.0.02021 & Fasciculus enameli & Enamel fascicle & Penacho del esmalte \\
\hline H3.04.01.0.02023 & Linea incrementalis enamelea & Enamel incremental line & Líneas incrementales del esmalte \\
\hline H3.04.01.0.02089 & Organum enameleum & Enamel organ & Órgano del esmalte \\
\hline H3.04.01.0.02093 & Epithelium enameleum externum & External enamel epithelium & Epitelio externo del esmalte \\
\hline H3.04.01.0.02094 & Reticulum enameleum stellatum & Stellate reticulum & Retículo estrellado del esmalte \\
\hline H3.04.01.0.02096 & $\begin{array}{c}\text { Epithelium enameleum } \\
\text { internum }\end{array}$ & Internal enamel epithelium & Epitelio interno del esmalte \\
\hline H3.04.01.0.05012 & Enameloblastus & Enameloblast & Enameloblasto \\
\hline H3.04.01.0.02097 & Cuticula enameli & Enamel cuticle & Cutícula del esmalte \\
\hline H3.04.01.0.02100 & $\begin{array}{l}\text { Epithelium enameleum } \\
\text { reductum }\end{array}$ & Reduced enamel epithelium & Epitelio reducido del esmalte \\
\hline H3.04.01.0.02103 & Junctio dentinoenamelea & $\begin{array}{l}\text { Enamel dentine junction; Dentin } \\
\text { enamel junction }\end{array}$ & Unión enamelo-dentinaria \\
\hline TE - 2605 & Organum enameleum & Enamel organ & Órgano del esmalte \\
\hline TE - 2606 & Lamina basalis enameli & Enamel basal lamina & Lámina basal del esmalte \\
\hline TE - 2607 & Nodus enameleus & Enamel knot & Nudo del esmalte \\
\hline TE - 2608 & Nodus enameleus primarius & Primary enamel knot & Nudo primario del esmalte \\
\hline TE - 2609 & $\begin{array}{c}\text { Nodus enameleus } \\
\text { secundarius }\end{array}$ & Secondary enamel knot & Nudo secundario del esmalte \\
\hline TE - 2614 & Chorda enamelea & Enamel cord & Cuerda del esmalte \\
\hline TE - 2616 & $\begin{array}{c}\text { Epithelium enameleum } \\
\text { externum }\end{array}$ & External enamel epithelium & Epitelio externo de esmalte \\
\hline 2617 & Reticulum stellatum & Stellate reticulum & Retículo estrellado \\
\hline 2618 & $\begin{array}{l}\text { Stratum enameleum } \\
\text { intermedium }\end{array}$ & Stratum intermedium & Estrato intermedio del esmalte \\
\hline 2619 & Epithelium enameleum internum & Internal enamel epithelium & Epitelio interno del esmalte \\
\hline 2624 & Enamelogenesis & Enamelogenesis & Enamelogenesis \\
\hline 2625 & Preenameloblastus & Pre-enameloblast & Pre-enameloblasto \\
\hline 2626 & Enameloblastus & Enameloblast & Enameloblasto \\
\hline 2629 & Prisma enameli & Enamel prism & Prisma del esmalte \\
\hline 2655 & $\begin{array}{c}\text { Epithelium enameleum } \\
\text { reductum }\end{array}$ & Reduced enamel epithelium & $\begin{array}{c}\text { Epitelio reducido del órgano } \\
\text { del esmalte }\end{array}$ \\
\hline 2657 & Vestigium epithelii enamelei & Rest of enamel epithelium & $\begin{array}{c}\text { Restos epiteliales del órgano } \\
\text { del esmalte }\end{array}$ \\
\hline 2676 & Enameloma & Enameloma & Enameloma \\
\hline 2682 & Opacitas enameli & Enamel opacity & Opacidad del esmalte \\
\hline 2683 & Extensio cervicalis enameli & Cervical enamel extension & Extensión cervical del esmalte \\
\hline 2696 & Hypoplasia enameli & Enamel hypoplasia & Hipoplasia del esmalte \\
\hline 2701 & Enamelogenesis imperfecta & Enamelogenesis imperfecta & Enamelogénesis imperfecta \\
\hline
\end{tabular}




\section{CONCLUSIONES}

El esmalte dental es un término encontrado dentro de Terminologia Anatomica, Histologica y Embryologica, siendo posible hallar sus palabras derivadas, en relación a tres raíces: amelo, adamantino y enamel, desde el latín oficial de terminología hasta su traducción a los idiomas vernáculo. En esta búsqueda fue posible corroborar que estos orígenes del término obedecen a su vez a diferentes idiomas, ya que adamantino tiene origen greco-latino, mientras que enamel tiene un origen más incierto, en relación a los idiomas anglonormando y antiguo alto germánico. Por este motivo, por la gran cantidad de evidencia científica indexada en torno a este término y dada la referencia a las características estéticas del esmalte dental, es que la propuesta realizada se enfoca en unificar la raíz común de las estructuras relacionadas en torno al enamel inglés, y su forma latinizada enamelum, abandonando las formas adamantinas de nombrar a esta región del diente. Dentro de las limitaciones de esta investigación, están las proyecciones en estudios moleculares relacionadas a este tejido, ya que existen moléculas, marcadores, y otros aspectos, nombrados a su vez en relación a su raíz griega adamantino.

PANES, C.; NICHOLSON, C. \& DEL SOL, M. Enamel in terminology concordance and proposal for anatomical, histological and embryological terminology. Int. J.Morphol., 38(3):670-676, 2020.

SUMMARY: Dental enamel is a structure considered in Anatomical, Histological and Embryological Terminology. The included terms related to it have three associated roots: enamel, amelo and adamantino. The objective of this article is to analyze the etymology of these roots and present proposals in Latin and Spanish that unify the denominations related to tooth enamel. In the search, medical, terminological and etymological dictionaries were used. The results obtained indicate three different origins for each root: adamantine is a Greco-Roman term related to the extreme hardness of a structure; enamel has an Anglo-Norman and ancient high Germanic origin related to the action of melting, melting, and the aesthetic appearance that this produces in a material; and amelo, which in itself is related to botany while the full term ameloblast, was coined by an American academic. Although the etymology of adamantine and enamel indicate characteristics of firmness and aesthetics, respectively, in the development of the proposal it was decided to maintain the Latinized term enamelum and its adjective enameleus, eliminating the other two roots related to dental enamel. The proposal was made for changes in the terms of the Latin language and its translation into Spanish, according to the requirements of the FIPAT for Anatomical, Histological and Embryological Terminology, in order to unify the denomination of the associated terms and derivatives of dental enamel , for academic and research purposes.

PALABRACLAVE: Esmalte; Terminologia Anatomica, Histologica y Embryologica.

\section{REFERENCIAS BIBLIOGRÁFICAS}

Algieri et. al. Análisis del Conocimiento de la Terminología Anatómica Internacional (TAI) por el Cirujano General. Int. J. Morphol., 31(4):1511-6, 2013.

Blánquez, A. Diccionario Latino-Español. Sopena, Barcelona, España, 1975.

Cortés, F. \& Ureña, J. Diccionario Médico-Biológico, Histórico y Etimológico. Salamanca, Ediciones Universidad de Salamanca, 2011. Disponible en: www.dicciomed.usal.es

Donkin, T.C. An Etymological Dictionary of the Romance Languages: Chiefly From the German of Friedrich Diez. Ed. Williams and Norgate, Londres, 1864.

Federative International Committee on Anatomical Terminology (FICAT). Terminologia Anatomica. International Anatomical terminology. Stuttgart, Georg Thieme Verlag, 2001.

FIPAT. Terminologia Anatomica. 2nd ed. FIPAT.library.dal.ca. Federative International Programme for Anatomical Terminology, 2019.

FIPAT. Terminologia Embryologica. 2nd ed. FIPAT.library.dal.ca. Federative International Programme for Anatomical Terminology, 2017.

Federative International Committee on Anatomical Terminology (FICAT). Terminologia Histologica: International Terms for Human Cytology and Histology. Philadelphia, Wolters Kluwer/Lippincott Williams \& Wilkins, 2008.

Hard, R. El Gran Libro de La Mitología Griega. Ed. La esfera de los libros, Madrid, España, 2009.

Hoad, T. Concise Dictionary of English Etymology. Oxford University Press, NY, 1996.

Lewis, T. C \& Short, C. Latin-English Lexicon, Ed. Clarendon Press, Oxford (1879). Disponible en: http://perseus.uchicago.edu/Reference/ lewisandshort.html

Navarrete, J. \& Roa, I. Uso de Terminologia Histologica en Textos de Histología Oral: Capítulo Esmalte.Int.J.Morphol., 36(3):1022-7, 2018.

Pantelia, M. Liddell-Scott-Jones Greek-English Lexicon. Disponible en: http://stephanus.tlg.uci.edu/lsj/\#eid=1

Partridge, E. A Short Etymologycal Dictionary of Modern English. 4 ed. Ed. Routledge, Londres, 1966.

Creane, G. Perseus Digital Librar (Internet). (Actualizado 2007; citado enero 2020). Disponible en: http://www.perseus.tufts.edu/hopper/

Real Academia Española. Diccionario de la lengua española (22a ed.), 2001. Consultado en http://www.rae.es/rae.html

Real Academia Nacional de Medicina. Diccionario de Términos Médicos. Panamericana. Madrid, 2011. Disponible en: dtme.ranm.es

Vásquez, B. \& del Sol, M. Terminologia Anatomica y Terminologia Histologica. Un Lugar de Encuentro entre los Morfólogos. Int. J. Morphol., 33(4):1585-90, 2015.

Vox. Diccionario Ilustrado Latino-Español, Español-Latino. 23a ed. Ed. Larousse, Barcelona, 2012.

Vox. Diccionario Manual Griego clásico-Español. Ed. Vox, 2006.

Direccion de correspondecia

Camila Panes

Programada de Doctorado en Ciencias Morfológicas

Facultad de Medicina

Universidad de La Frontera

Temuco - CHILE

Email:camilapanesv@gmail.com

Recibido : 10-11-2019

Aceptado: $28-01-2020$ 